\title{
Comissões de Controle de Infecção Hospitalar: perspectiva de ações do Conselho Regional de Enfermagem
}

Hospital Infection Control Committees: perspective of actions of the Regional Council of Nursing

Comisiones de Control de Infección Hospitalaria: perspectiva de acciones del Consejo Regional de Enfermería

RESUMO. Objetivo: Caracterizar as Comissões de Controle de Infecção Hospitalar (CCIH) nos hospitais, por meio da análise dos relatórios das fiscalizações do Conselho Regional de Enfermagem (Coren-SP); obter subsídios para proposta de melhoria nas atividades de enfermeiros das $\mathrm{CClH}$ e aprimorar as ações de fiscalização. Metodologia: Estudo transversal descritivo e exploratório, por meio de consulta dos relatórios de fiscalizações realizadas entre setembro de 2012 e fevereiro de 2017. Resultados: Foram avaliados relatórios de todas as instituições hospitalares $(n=838)$ no Estado de São Paulo. Dessas, 778 instituições (92,84\%) possuíam $\mathrm{CClH}$; porém em 318 (40,87\%), o enfermeiro não era exclusivo, realizando ações gerenciais ou assistenciais ao mesmo tempo em que atuava na $\mathrm{CCIH}$, descumprindo a legislação vigente. Das 60 instituições que não possuíam $\mathrm{CCIH}, 51$ $(85,0 \%)$ eram de pequeno porte ( $\geq 50$ leitos). Conclusão: A falta de conformidade com os requisitos legais em recursos humanos sugere que a estrutura de prevenção de infecções relacionadas à assistência à saúde (IRAS) ainda é deficiente em muitos hospitais do estado de São Paulo, o pode levar a falhas na implementação efetiva de medidas de prevenção. Auditorias e fiscalizações, como as realizadas pelo Coren-SP desempenham papel importante e podem fazer parte de uma ampla estratégia governamental para alcançar melhorias nos serviços de saúde.

Palavras-chave: Infecção Hospitalar. Programa de Controle de Infecção Hospitalar. Regulação e Fiscalização em Saúde.

ABSTRACT. Objective: Characterize the Commission for the Control of Hospital Infections $(\mathrm{CCHI})$ in hospitals by means of the analysis of inspection reports of the Regional Council of Nursing (Coren-SP); obtain subsidies to propose improvements in $\mathrm{CCHI}$ nursing activities and improve inspection actions. Methodology: Descriptive and exploratory cross-sectional study by consulting inspection reports from September 2012 to February 2017. Results: Reports from all hospitals in the state of São Paulo were evaluated $(n=838)$. Of these, 778 hospitals (92.84\%) had CCHI, but in 318 (40.87\%), nurses were not exclusive, carrying out managerial or assistance actions while acting in the $\mathrm{CCHI}$, not complying with current

\footnotetext{
${ }^{1}$ Mestre em Ciências da Saúde. Fiscal. Conselho Regional de Enfermagem do Estado de São Paulo. São Paulo. Email: luizlamblet7@gmail.com

2 Doutora em Ciências Médicas. Professora Doutora, do Departamento de Enfermagem em Saúde Coletiva da Escola de Enfermagem da Universidade de São Paulo. Universidade de São Paulo. Escola de Enfermagem. São Paulo. Email: padoveze@usp.br
} 
legislation. Of the 60 institutions that didn't have CCHI, 51 (85.0\%) were small-sized $(\geq 50$ beds). Conclusion: The lack of compliance with legal requirements in human resources suggests that the healthcare-associated infection $(\mathrm{HCl})$ prevention structure is still deficient in many hospitals in the state of São Paulo, which may lead to failures in the effective implementation of prevention measures. Audits and inspections, such as those conducted by Coren-SP, play an important role and may become part of a broader governmental strategy to achieve improvements in health services.

Keywords: Cross Infection. Hospital Infection Control Program. Health Care Coordination and Monitoring.

RESUMEN. Objetivo: Caracterizar las Comisiones de Control de Infección Hospitalaria $(\mathrm{CCIH})$ en los hospitales por medio del análisis de los informes de las fiscalizaciones del Consejo Regional de Enfermería (Conselho Regional de Enfermagem - Coren-SP); obtener subsidios para propuesta de mejoría en las actividades de los enfermeros de las $\mathrm{CCIH} \mathrm{y}$ perfeccionar las acciones de fiscalización. Metodología: Estudio transversal descriptivo y exploratorio por medio de consulta de los informes de fiscalizaciones realizadas entre setiembre de 2012 y febrero de 2017. Resultados: Fueron evaluados los informes de todas las instituciones hospitalarias $(n=838)$ en el Estado de São Paulo. De esas, 778 instituciones $(92,84 \%)$ tenían $\mathrm{CCIH}$; pero en 318 (40,87\%), el enfermero no era exclusivo, realizando acciones de gestión administrativa o asistenciales al mismo tiempo en que actuaba en la $\mathrm{CCIH}$, trasgrediendo la legislación vigente. De las 60 instituciones que no tenían $\mathrm{CCIH}, 51$ $(85,0 \%)$ eran de pequeño porte ( $\geq 50$ lechos). Conclusión: La falta de conformidad con los requisitos legales en recursos humanos sugiere que la estructura de prevención de infecciones relacionadas a asistencia en salud (IRAS) aún es deficiente en muchos hospitales del estado de São Paulo, o puede acarrear fallas en la implementación efectiva de medidas de prevención. Auditorías y fiscalizaciones, como las realizadas por Coren-SP desempeñan un papel importante y pueden formar parte de una amplia estrategia gubernamental para alcanzar mejorías en los servicios de salud.

Palabras-Ilave: Infección Hospitalaria. Programa de Control de Infecciones Hospitalarias. Regulación y Fiscalización en Salud.

\section{Introdução}

As normativas oficiais brasileiras impõem grande responsabilidade ao enfermeiro no que tange às ações de prevenção das Infecções Relacionadas à Assistência a Saúde (IRAS). Em primeiro lugar, a Lei do Exercício Profissional de Enfermagem atribui ao enfermeiro à responsabilidade de atuar em conjunto com a equipe de saúde para "prevenção e controle sistemático da infecção hospitalar" (1). Específica para a área de atuação, a Portaria no 2.616/1998 (2) estabelece diretrizes e normas para a prevenção e o controle das IRAS e indica a participação preferencialmente de um enfermeiro entre os membros executores dessas diretrizes. Além destas, o Código de Defesa do Consumidor (CDC) estabelece como dever do Estado garantir a saúde por meio de políticas econômicas e 
sociais que visem reduzir risco de doenças e agravos. Diante disto, a participação do enfermeiro no processo assistencial e político é relevante (3).

Ademais, segundo o Código Civil Brasileiro, aquele que por ação ou omissão voluntária, negligência ou imprudência, violar direito e causar dano à outra pessoa, mesmo que moral comete ato ilícito (4). Entre os profissionais de enfermagem essas responsabilidades recaem com peso maior sobre o profissional enfermeiro, pois segunda a legislação que regulamenta as atribuições da enfermagem, além das responsabilidades diretas sobre suas ações, compete ao enfermeiro coordenar as ações dos demais profissionais da equipe (5).

O desconhecimento das legislações não exime 0 enfermeiro de suas responsabilidades. O conhecimento dos direitos e deveres éticos e legais é necessário para o adequado cumprimento das obrigações profissionais (6). Por meio das leis se criam e extinguem direitos e deveres; se protegem direitos dos pacientes e dos profissionais de saúde; e pela participação dos profissionais de enfermagem no aprimoramento das legislações vigentes se dá o empoderamento da categoria (7). Do ponto de vista ético, uma das principais responsabilidades do profissional de enfermagem é assegurar ao paciente, família e comunidade uma assistência livre de danos por imperícia, imprudência ou negligência, bem como protegê-los dessas situações (8).

A atuação do enfermeiro na prevenção de IRAS torna-se complexa sob o ponto de vista ético e legal devido à escassez de recursos humanos, falta de financiamento específico para IRAS, dimensão territorial brasileira com locais de difícil acesso, grande número de instituições e muitos hospitais pequenos com dificuldades para instituir um programa de prevenção de IRAS, oferta assistencial heterogênea e insuficiência de leitos de terapia intensiva (9). De acordo com a legislação vigente, todo hospital brasileiro deve formar uma Comissão de Controle de Infecção Hospitalar (CCIH) para a implantação de um Programa de Controle de Infecções Hospitalares (PCIH). A abrangência deste programa não se resume somente em implantar a $\mathrm{CClH}$ ou nomear os profissionais participantes, mas sim estabelecer um conjunto de ações cuja qualidade somente poderá ser evidenciada pela construção de indicadores e avaliação das atividades realizadas (10). O papel do enfermeiro no PCIH é fundamental desde sua atividade assistencial, de vigilância e de produção dos indicadores, bem como na área de gestão. Extramuros, o enfermeiro pode ser um agente 
social envolvido nas políticas de saúde e na proposição de reformulação das legislações vigentes (7).

A atividade-fim do sistema Conselho Federal/Conselhos Regionais de Enfermagem é a fiscalização do exercício profissional (11), com foco educativo e participativo (12). Os procedimentos de fiscalização do Conselho Regional de Enfermagem de São Paulo (Coren$\mathrm{SP}$ ) descritos em documentos normativos operacionais determinam que o fiscal caracterize a instituição de saúde e o serviço de enfermagem. Dentre os itens levantados por meio de um roteiro de fiscalização, é requerido verificar a existência de $\mathrm{CCIH}$ e a presença de enfermeiro na composição dessa equipe. Na inexistência de enfermeiro na $\mathrm{CCIH}$, o fiscal deverá recomendar o disposto na Portaria GM/MS n² 2.616/1998 e descrever as orientações fornecidas em relatório circunstanciado. Compreende-se da atuação do Coren-SP em relação às ações de fiscalização do exercício profissional a busca do cumprimento das legislações de enfermagem vigentes, educação sobre o papel ético e legal do profissional e promoção do empoderamento do enfermeiro como agente promotor de mudança nas políticas do sistema de saúde.

Este trabalho se justifica pela necessidade de fortalecimento do papel do enfermeiro dentro das instituições de saúde. Em relação à prevenção de IRAS, engloba três níveis de atuação dos enfermeiros: na área assistencial, na área de gestão e nas ações como enfermeiro da $\mathrm{CClH}$. O enfermeiro assistencial é o principal agente para efetivação das estratégias de prevenção de IRAS, pois no seu espaço de atuação viabiliza e concretiza as práticas institucionais. O enfermeiro gestor estabelece ou deveria ter o empoderamento para estabelecer as estratégias de prevenção de IRAS, por meio do direcionamento dos recursos humanos, financeiros e materiais às ações de prevenção das IRAS. O enfermeiro da CCIH é aquele que realiza levantamento de dados e orientações às equipes multiprofissionais sobre os dados epidemiológicos institucionais das IRAS. Todos esses papéis são interrelacionados e sinérgicos. Por este motivo, o reconhecimento da condição atual da inserção dos enfermeiros nas $\mathrm{CCIH}$ é o primeiro passo para o delineamento de diretrizes de melhoria para a atuação dos enfermeiros nessas comissões.

O objetivo desta pesquisa foi caracterizar o cenário das $\mathrm{CCIH}$ em todas as instituições hospitalares fiscalizadas pelo Coren-SP, na perspectiva da avaliação fiscalizatória. 


\section{Metodologia}

Foi realizado estudo transversal descritivo e exploratório, por meio de consulta de dados secundários obtidos junto ao Coren-SP.

A pesquisa foi realizada no âmbito do Estado de São Paulo. Foram consideradas as instituições classificadas como "hospital". O Coren-SP possui um modelo de fiscalização seguindo um roteiro padronizado de itens mínimos a serem levantados nas ações de fiscalização que incluem questões relacionadas à $\mathrm{CCIH}$, os quais alimentam um banco de dados específico. Esse roteiro foi elaborado e implantado após consulta com participação de todos os fiscais do Coren-SP do estado de São Paulo, por meio de reuniões, revisões e sugestões de legislações a serem incluídas e foi implantado a partir de setembro de 2012.

As informações foram obtidas por meio de busca no banco de dados eletrônico do Coren-SP, referente ao período de setembro de 2012 a fevereiro de 2017. O setor de tecnologia da informação (GTI) forneceu a listagem das instituições cadastradas no sistema como "hospital". Ao total constavam 979 instituições. Foram analisados todos os relatórios das fiscalizações realizadas no período supracitado. Uma vez que muitos estabelecimentos foram auditados mais do que uma vez, foram incluídos no estudo apenas dados do relatório mais recente de fiscalização de cada instituição hospitalar, objetivando a análise das informações mais atualizadas. Para a coleta de dados, os relatórios foram lidos individualmente para localização das informações necessárias no corpo do texto elaborado pelos fiscais. Os dados foram tabulados em planilha Excel®. As variáveis coletadas foram: caracterização da instituição hospitalar (pública, privada ou filantrópica); localização (município e se na cidade de São Paulo, qual o bairro/região); número de leitos gerais; número de leitos de terapia intensiva (adulta, pediátrica ou neonatal); leitos de pronto socorro ou pronto atendimento; caracterização da $\mathrm{CCIH}$ (existência ou inexistência de enfermeiro na $\mathrm{CCIH}$ ); orientações dadas pelos fiscais sobre a Portaria no 2616/98; e número de profissionais de enfermagem por categoria (enfermeiros, auxiliares e técnicos de enfermagem).

De fevereiro a julho de 2017 foi realizada a análise dos dados. Os dados foram interpretados frente à sua relevância na contribuição para apontar estratégias para fortalecer o papel do enfermeiro nas ações de prevenção de IRAS e no aspecto ético e profissional perante as instituições. 
Foi solicitada autorização ao Coren-SP, protocolada em 25 de novembro de 2015, para coleta de dados que foi deferida em 08 de dezembro de 2015. Após essa autorização prévia, o projeto foi submetido ao Comitê de Ética em Pesquisa da Escola de Enfermagem da Universidade de São Paulo - EFE-USP sob número C.A.A.E. ㄲo 52041515.0.0000.5392 e foi emitida aprovação em 01/02/2016. Após aprovação do projeto, foi solicitada autorização para efetivação da pesquisa e coleta de dados ao Coren-SP por meio de Processo Administrativo no 001220/2016. A Diretoria do Coren-SP em sua 1105a Reunião Ordinária de 23/02/2016, apreciou o Parecer Consubstanciado do CEP e aprovou por unanimidade a realização da pesquisa.

\section{Resultados}

Dentre as instituições previamente selecionadas, 141 relatórios não foram incluídos, pelos seguintes motivos: 20 tiveram fiscalização anterior a setembro de 2012; 31 apresentavam duplicidade de código no sistema eletrônico do Coren-SP; 28 eram serviços ambulatoriais, hospital-dia, pronto-socorro, serviços administrativos ou setor terceirizado dentro da instituição hospitalar; 32 eram códigos desativados do sistema eletrônico do Coren-SP; e em 30 não havia sido realizada fiscalização em nenhum momento. Dessa maneira, foram incluídos para análise relatórios de fiscalização de 838 instituições de um total de 979 classificadas como hospitalares no sistema eletrônico do Coren-SP.

O Estado de São Paulo possui 17 Departamentos Regionais de Saúde (DRS) com finalidade administrativa. O Conselho Regional de Enfermagem de São Paulo (Coren-SP), sendo uma autarquia federal, possui uma divisão administrativa determinada por meio de atos deliberativos de sua Plenária. Não há concordância plena entre as divisões administrativas das DRS e do Coren-SP e para o estudo optou-se em utilizar as divisões administradas conforme este último.

Verificou-se que em $92,84 \%$ das instituições hospitalares do estado de São Paulo há constituída formalmente uma $\mathrm{CCIH} \mathrm{(778/838).} \mathrm{As} \mathrm{subseções} \mathrm{com} \mathrm{menor} \mathrm{percentual} \mathrm{foram:}$ Marília, Santos e Botucatu, com respectivamente 18,64\%, 15,15\% e 12,50. As regiões com maior percentual foram: Guarulhos, Ribeirão Preto e Araçatuba que apresentaram respectivamente 100\%, 97,85\% e 97,44\%. A sede (São Paulo) e Campinas possuem o maior número de instituições com frequência de aproximadamente $92 \%$ de presença de $\mathrm{CCIH}$ (tabela 1). De modo geral, no Estado predominam hospitais de médio $(39,98 \%)$ e de 
pequeno porte (38,07\%). Do total de 11 hospitais com mais de 501 leitos, 8 estão na Sede (72,73\%), sendo os outros 3 nas subseções de Ribeirão Preto (9,09\%), São José do Rio Preto $(9,09 \%)$ e Santos $(9,09 \%)$.

Tabela 1 - Distribuição de frequência das Comissões de Controle de Infecções Hospitalares (CCIH) nas instituições hospitalares, segundo as subseções. São Paulo, 2017.

\begin{tabular}{lccc}
\hline \multicolumn{1}{c}{ Subseção } & sim & CCIH \\
\hline Matriz & $\mathrm{n}$ & sim & 93,06 \\
Campinas & 116 & 201 & 92,24 \\
Ribeirão Preto & 93 & 91 & 97,85 \\
São José do Rio Preto & 54 & 50 & 92,59 \\
Marília & 59 & 48 & 81,36 \\
São José dos Campos & 51 & 48 & 94,12 \\
Santo André & 42 & 40 & 95,24 \\
Araçatuba & 39 & 38 & 97,44 \\
Botucatu & 40 & 35 & 87,50 \\
Guarulhos & 35 & 35 & 100,00 \\
Presidente Prudente & 32 & 30 & 93,75 \\
Santos & 33 & 28 & 84,85 \\
Itapetininga & 28 & 27 & 96,43 \\
\hline Total & 838 & 778 & 92,84 \\
\hline
\end{tabular}

Fonte: dados secundários do sistema do Coren-SP.

Dentre as 60 instituições sem CCIH constituída, 45 (75,0\%) eram privadas e 15 (25,0\%) eram públicas. Entre os hospitais de pequeno porte $(n=319), 51$ não possuíam CCIH constituída num total de $85,00 \%$ (51/60) (tabela 2).

Tabela 2 - Distribuição de instituições hospitalares com Comissões de Controle de Infecções Hospitalares $(\mathrm{CClH})$ pelo porte e tipo de instituição no Estado de São Paulo. São Paulo, 2017.

\begin{tabular}{lccccc}
\hline & & \multicolumn{2}{c}{ CCIH } & \multicolumn{2}{c}{ Público } \\
Porte & $\mathrm{N}$ & Sim & $\%$ & Sim & $\%$ \\
\hline Pequeno Porte $^{*}$ & 319 & 228 & 71,47 & 40 & 12,54 \\
Médio Porte+ & 335 & 264 & 78,81 & 64 & 19,10 \\
Grande Porte \pm & 173 & 106 & 61,27 & 65 & 37,57 \\
Extra Grande $\S$ & 11 & 8 & 72,73 & 3 & 27,27 \\
\hline Total & 838 & 606 & 72,32 & 172 & 20,53 \\
\hline
\end{tabular}

*PP - até 50 leitos; +MP - de 51 a 150 leitos; $\pm G P$ - de 151 a 500 leitos; §EG - acima de 501 leitos.

Fonte: dados secundários do sistema do Coren-SP. 
Das 778 instituições hospitalares que possuíam CCIH constituída, 416 (53,47\%) mantinham enfermeira exclusiva para as atividades de controle de IRAS. Verificou-se que em 40 relatórios não foi informado se havia enfermeiro exclusivo no $\mathrm{CCIH}(5,14 \%)$. Em 2 instituições foi informado que havia $\mathrm{CCIH}$, porém com vaga aguardando contratação. Em outras 2 instituições havia atuação de profissional auxiliar de enfermagem atuando na $\mathrm{CCIH}$, sem enfermeiro. Em 318 instituições (40,87\%), a enfermeira não realizava atividades exclusivas de $\mathrm{CCIH}$, acumulando atividades assistenciais em 198 instituições (62,26\%) e em 120 atividades gerenciais (37,73\%). Segundo o levantamento dos relatórios de fiscalização do Coren-SP (tabela 3), há 40.721 enfermeiros atuando em instituições hospitalares, sendo $66,22 \%$ (29.967) em instituições privadas e 33,78\% (13.754) em públicas. Dos técnicos de enfermagem, $56.030(75,04 \%)$ e dos auxiliares de enfermagem, $46.174(60,08 \%)$ atuam em instituições privadas. 
Tabela 3 - Distribuição de profissionais de enfermagem por categoria profissional de acordo com o tipo e porte de instituição hospitalar no Estado de São Paulo. São Paulo, 2017

\begin{tabular}{|c|c|c|c|c|c|c|c|c|c|c|}
\hline \multirow{3}{*}{ Categoria } & \multirow{3}{*}{ Tipo instituição } & \multirow{3}{*}{ Total } & \multicolumn{8}{|c|}{ Porte } \\
\hline & & & \multicolumn{2}{|c|}{ Pequeno } & \multicolumn{2}{|c|}{ Médio } & \multicolumn{2}{|c|}{ Grande } & \multicolumn{2}{|c|}{ Extra Grande } \\
\hline & & & $\mathrm{n}$ & $\%$ & $\mathrm{n}$ & $\%$ & $\mathrm{n}$ & $\%$ & $\mathrm{n}$ & $\%$ \\
\hline \multirow{3}{*}{ Enfermeiros } & Pública & 13754 & 760 & 5,52 & 3244 & 23,59 & 8319 & 60,48 & 1431 & 10,40 \\
\hline & Privada & 26967 & 3155 & 11,70 & 8282 & 30,71 & 11792 & 43,73 & 3738 & 13,86 \\
\hline & Total & 40721 & 3915 & 9,61 & 11526 & 28,30 & 20111 & 49,39 & 5169 & 12,69 \\
\hline \multirow{3}{*}{ Técnicos } & Pública & 18635 & 1243 & 6,67 & 4475 & 24,01 & 10493 & 56,31 & 2424 & 13,01 \\
\hline & Privada & 56030 & 6693 & 11,95 & 19499 & 34,80 & 25469 & 45,46 & 4369 & 7,80 \\
\hline & Total & 74665 & 7936 & 10,63 & 23974 & 32,11 & 35962 & 48,16 & 6793 & 9,10 \\
\hline \multirow{3}{*}{ Auxiliares } & Pública & 30684 & 1670 & 5,44 & 7452 & 24,29 & 19787 & 64,49 & 1775 & 5,78 \\
\hline & Privada & 46174 & 3981 & 8,62 & 14822 & 32,10 & 19776 & 42,83 & 7595 & 16,45 \\
\hline & Total & 76858 & 5651 & 7,35 & 22274 & 28,98 & 39563 & 51,48 & 9370 & 12,19 \\
\hline
\end{tabular}

* Porte: Pequeno - até 50 leitos; Médio - de 51 a 150 leitos; Grande - de 151 a 500 leitos; Extra Grande - acima de 501 leitos.

Fonte: Dados secundários do sistema do Coren-SP. 


\section{Discussão}

Os dados obtidos por meio da consulta do sistema do Coren-SP, permitiram identificar que na maioria das regiões a presença de hospitais com $\mathrm{CCIH}$ constituída excede a 90\% das instituições incluídas no estudo. Não obstante algumas regiões com valores inferiores, estas se encontram acima de $80 \%$ de presença da $\mathrm{CCIH}$. Este número, embora pareça positivo, ainda se apresenta aquém da expectativa, uma vez que o valor esperado seria 100\%, que só foi observado na região de Guarulhos. Este dado revela uma fragilidade no cumprimento do que é estabelecido pelas normativas legais e aponta para a necessidade de medidas enérgicas por parte dos órgãos competentes para reverter este cenário.

Um recente estudo brasileiro avaliou a prevalência de IRAS (13) e outro as condições estruturais dos PCIH em uma amostra de 152 hospitais brasileiros públicos e privados (14). Este revelou a prevalência de IRAS distribuídas igualmente entre as diferentes instituições, com taxa geral de $10,8 \%$, sendo que em hospitais de grande porte a taxa geral foi de $13,5 \%$ (13). Com relação à infraestrutura necessária para prevenção de IRAS no Brasil, foram evidenciados menores índices de conformidade aos PCIH entre hospitais com até 50 leitos (14); entretanto o estudo não particularizou dados por Estado. Uma avaliação fiscalizatória em 2008 em uma amostra de 158 hospitais pelo Conselho Regional de Medicina e Ministério Público do Estado de São Paulo apontou que mais de $90 \%$ dos avaliados não cumpria pelo menos uma das exigências da legislação na área de prevenção e controle de IRAS (15). O estudo foi publicado em 2010 com detalhamento das informações, porém sem esclarecer os critérios de definição da amostra. Um estudo realizado com 50 hospitais no Estado do Paraná apontou a conformidade geral obtida nos PCIH de 71,0\% com desvio padrão de 23,8 (16). Contudo, a comparação entre os estudos da literatura e os resultados obtidos em nosso estudo não pode feita diretamente devido a diferenças nos métodos empregados.

No estudo, os dados levantados na fiscalização de que 51 hospitais de pequeno porte (com até 50 leitos) não possuíam $\mathrm{CClH}$ constituída confirmam a necessidade de avaliar as dificuldades que essas instituições enfrentam para estruturar a $\mathrm{CCIH}$. A informação de que a legislação vigente permite o consórcio entre hospitais para a implantação do $\mathrm{PCIH}(2)$ pode ser uma estratégia importante no auxílio aos gestores de instituições com recursos financeiros e humanos limitados.

Observou-se que as instituições que não possuíam $\mathrm{CClH}$ eram na sua maioria de caráter privado. Contudo, este dado pode apenas refletir a distribuição de gestão privada ou 
pública no estado de São Paulo. Entretanto, este dado aponta para a necessidade de estudos adequadamente delineados que permitam identificar se existe associação entre o tipo de gestão administrativa e a presença e qualificação das $\mathrm{CCIH}$.

Este estudo evidenciou que as instituições privadas também apresentam maior proporção de enfermeiros com funções gerenciais ou assistenciais que também atuam nas $\mathrm{CCIH}$ dos seus hospitais ( $\mathrm{n}=279 ; 87,5 \%$ ), conforme declarado nas fiscalizações. Esse dado reforça que somente a legislação que exige a implantação de $\mathrm{CCIH}$ não é suficiente para garantir a qualidade desses serviços.

A prevenção das IRAS é problema complexo e multifatorial que exige grandes esforços e empenho para que resultados positivos sejam alcançados (17). O sistema de saúde brasileiro apresenta uma estruturação ampla, que engloba parceria pública e privada, legislações específicas e grupos técnico-científicos atuantes, sendo que o grande ponto crítico em relação às IRAS nesse contexto parece ser a falta de um foco político como garantidor de financiamento das ações relevantes à qualificação dos serviços (18). A estruturação de um sistema de vigilância de IRAS no Brasil que permita a comparação de dados institucionais entre as diversas regiões e tipos de instituições e que forneça subsídios para ações governamentais de abrangência nacional é essencial (19). A eficácia desse sistema depende de estruturação adequada dessa rede de vigilância de IRAS (20). No enfoque da prevenção de IRAS, a fiscalização pode e deve avançar tanto na ampliação das fiscalizações como na qualificação dos fiscais (21). As condições para prevenção das IRAS estão relacionadas a fatores sociais, financeiros, políticos e educacionais e as ações do enfermeiro nessa área serão mais efetivas quanto maior seu entendimento e envolvimento nessas esferas de atuação (22). As parcerias entre órgãos regulatórios podem permitir avanços de qualidade, resultando em benefícios para toda a sociedade (23).

É importante também destacar a necessidade de associar informações estruturais com resultados e processos, o que não foi objetivo neste estudo. Nesse levantamento foram verificados aspectos quantitativos de $\mathrm{CCIH}$, porém o estado de São Paulo possui um sistema de vigilância com levantamento de indicadores de resultados nas instituições (24). Além disso, também há indicadores de avaliação de estrutura técnico-operacional de $\mathrm{PCIH}$ validados (25) que podem ser aplicados em quaisquer instituições de saúde. A sinergia advinda da presença do fiscal de enfermagem na instituição vistoriando presencialmente 
toda instituição hospitalar em parceria com uma estratégia conjunta com a Secretaria de Estado da Saúde poderia resultar em ações de grande impacto.

A partir da análise dos dados epidemiológicos disponíveis no sistema Coren-SP esperou-se caracterizar as $\mathrm{CCIH}$ dos hospitais, a inserção dos enfermeiros nas ações de prevenção das IRAS e a atuação do Coren-SP nessa dinâmica, o que permitirá a elaboração de uma proposta de intervenção nos diversos âmbitos de atuação dos enfermeiros visando a melhoria do trabalho e do atendimento de saúde da população.

\section{Conclusões}

Os resultados obtidos neste estudo permitiram evidenciar que no Estado de São Paulo ainda há barreiras para a efetiva aplicação das normativas legais. A falta de conformidade com os requisitos legais em recursos humanos sugere que a estrutura de prevenção de IRAS ainda é deficiente em muitos hospitais do estado de São Paulo, o que pode levar a falhas na implementação efetiva de medidas de prevenção. Sugere-se como busca para soluções a alternativa de firmar parcerias como oportunidade singular de qualificar as instituições hospitalares em suas múltiplas responsabilidades. Auditorias e fiscalizações, como as realizadas pelo Coren-SP desempenham papel importante e podem fazer parte de uma ampla estratégia governamental para alcançar melhorias nos serviços de saúde.

\section{Referências}

1. Brasil. Lei $n^{\circ} 7.498$, de 25 de junho de 1986. Dispõe sobre a regulamentação do exercício da enfermagem, e dá outras providências. Diário Oficial da União, 26 jun 1986; Seção 1:1.

2. Ministério da Saúde. Portaria oㅡ 2.616, de 12 de maio de 1998. Dispõe sobre diretrizes e normas para a prevenção e o controle das infecções hospitalares. Diário Oficial da União, 13 mai 1998; Seção 1:133-5.

3. Ministério da Saúde. Lei oo 8.080 de 19 de setembro de 1990. Dispõe sobre as condições para a promoção, proteção e recuperação da saúde, a organização e o funcionamento dos serviços correspondentes e dá outras providências. Disponível em: http://www.planalto.gov.br/ccivil 03/leis/L8080.htm. [Acesso em 13.jul.2017]

4. Brasil. Lei ํㅜ 10.406, de 10 de janeiro de 2002. Institui o Código Civil. Disponível em: http://www.planalto.gov.br/ccivil 03/leis/2002/L10406.htm. [Acesso em 17.jul.2017] 
5. Brasil. Decreto no 94.406, de 08 de junho de 1987. Regulamenta a Lei no 7498, de 25 de junho de 1986, que dispõe sobre o exercício da Enfermagem, e dá outras providências. Diário Oficial da União, 9 jun 1987; Seção 1:8853.

6. Rosenstock KI, Soares MJ, Santos SR, Ferreira AS. Aspectos éticos no exercício da enfermagem: revisão integrativa da literatura. Cogitare Enferm. 2011; 16(4):727-33.

7. Oguisso T, Schimidt MJ, Freitas GF. Fundamentos teóricos e jurídicos da profissão de enfermagem. Enferm Foco. 2010; 1(1):9-13.

8. Conselho Federal de Enfermagem. Resolução n 11, de 08 de fevereiro de 2007. Aprova a reformulação do Código de Ética dos Profissionais de Enfermagem. Disponível em: https://www.diariodasleis.com.br/busca/exibelink.php?numlink=1-39-34-2007-02-09311. [Acesso em 13.jul.2017]

9. Padoveze MC, Fortaleza CM. Healthcare-associated infections: challenges to public health in Brazil. Rev Saúde Pública. 2014;48(6):995-1001.

10. Agência Nacional de Vigilância Sanitária. Programa Nacional de Prevenção e Controle de Infecções Relacionadas à Assistência à Saúde (2016-2020). Brasília: Anvisa; 2016.

11. Brasil. Lei $n^{0} 5.905$, de 12 de julho de 1973. Dispõe sobre a criação dos conselhos federal e regionais de enfermagem e das outras providências. Disponível em: http://www.cofen.gov.br/lei-n-590573-de-12-de-julho-de-1973 4162.html. [Acesso em 13.jul.2017]

12. Conselho Federal de Enfermagem. Resolução $n^{\circ}$ 374, de 23 de março de 2011. Normatiza o funcionamento do sistema de fiscalização do exercício profissional da enfermagem e dá outras providências. Disponível em: http://www.cofen.gov.br/resoluocofen-n-3742011 6590.html. [Acesso em 13.jul.2017]

13. Padoveze MC, Fortaleza CM, Kiffer C, Barth AL, Carneiro IC, Giamberardino HI, et al. Structure for prevention of health care-associated infect in Brazilian hospitals: a countrywide study. Am J Infection Control. 2016; 44(1):74-9.

14. Fortaleza CM, Padoveze MC, Kiffer CR, Barth AL, Carneiro IC, Giamberardino HI, et al. Multi-state survey of healthcare-associated infections in acute care hospitals in Brazil. $J$ Hosp Infect. 2017; 96(2):139-44.

15. Conselho Regional de Medicina do Estado de São Paulo (CREMESP). Ministério Público do Estado de São Paulo (MPSP). O Controle da Infecção Hospitalar no Estado de São Paulo. São Paulo: Conselho Regional de Medicina do Estado de São Paulo, 2010.

16. Alves $\mathrm{DCl}$, Lacerda RA. Evaluation of Programs of Infection Control related to Healthcare Assistance in Hospitals. Rev Esc Enferm USP. 2015; 49 (Esp):65-7. 
17. Paim J, Travassos C, Almeida C, Bahia L, Macinko J. The brazilian health system: history, advances and challenges. Lancet. 2011; 377(9779):1778-97.

18. Nogueira Junior C, Padoveze MC, Lacerda RA. Governmental surveillance system of healthcare-associated infection in Brazil. Rev Esc Enferm USP. 2014; 48(4):656-61.

19. Oliveira HM, Silva CP, Lacerda RA. Policies for control and prevention of infections related to healthcare assistance in Brazil: a conceptual analysis. Rev Esc Enferm USP. 2016; 50(3):505-11.

20. Nogueira-Junior C, Mello DS, Padoveze MC, Boszczowski I, Levin AS, Lacerda RA. Characterization of epidemiological surveillance systems for healthcare-associated infections (HAI) in the world and challenges for Brazil. Cad Saúde Pública. 2014; 30(1):11-20.

21. Costa EO, Medeiros SM, Germano RM. A fiscalização do exercício profissional no Conselho Federal de Enfermagem. REME Rev Min Enferm. 2014 ;18(1): 208-17.

22. Fontana RF, Lautert L. Aspectos ético-legais do controle da infecção hospitalar: algumas reflexões relativas ao enfermeiro. Cienc Cuid Saude. 2008; 7(4):546-50.

23. Puccini PT. Perspectivas do controle da infecção hospitalar e as novas forças sociais em defesa da saúde. Ciênc Saúde Coletiva. 2011; 16(7):3043-9.

24. Padoveze MC, Assis DB, Freire MP, Madalosso G, Ferreira SA, Valente MG, et al. Surveillance Programme for Healthcare Associated Infections in the State of São Paulo, Brazil. Implementation and the first three years' results. J Hosp Infect. 2010; 76(4):311-5.

25. Silva CP, Lacerda RA. Validation of a proposal for evaluating hospital infection control programs. Rev Saúde Pública. 2011;45(1):121-8.

\section{Como citar este artigo:}

Lamblet LCR, Padoveze MC. Comissões de Controle de Infecção Hospitalar: perspectiva de ações do Conselho Regional de Enfermagem. Revista Cadernos Ibero-Americanos de Direito Sanitário. 2018 jan./mar, $7(1): 29-42$. 